\title{
LAS INTERACCIONES ENTRE EL DERECHO ESTATAL Y LA CERTIFICACION COMERCIO JUSTO FAIRTRADE: GARANTIA DE UNA MEJOR PROTECCION DE LOS DERECHOS DE LOS TRABAJADORES Y DEL MEDIO AMBIENTE ${ }^{*}$
}

Marie-Claude Desjardins** Recibido: Abril 17 de 2015 Aprobado: Junio 1 de 2015

\section{RESUMEN}

El régimen de certificación Comercio Justo Fairtrade International es un modelo de certificación de RSE interesante por su interacción con el derecho estatal porqué sus disposiciones y su funcionamiento favorecen una mejor aplicación. La referencia al derecho estatal es un medio de incorporarlo al corpus normativo de la certificación Comercio Justo Fairtrade. La reiteración de la obligación que tienen los productores de respetar la legislación nacional constituye igualmente un medio de asegurar que la certificación no tenga como consecuencia una nivelación a la baja de las exigencias aplicables a los productores. La referencia a la legislación y a las prácticas nacionales permite, inversamente, no imponer tampoco obligaciones desproporcionadas a los productores. $\mathrm{Al}$ insistir sobre la necesidad de deber conformarse tanto a las normas surgidas de los estándares como a aquellas que provienen del derecho estatal, se favorece igualmente un mejor conocimiento del derecho estatal. A pesar de estas ventajas, la incorporación de normas de derecho nacional es sin embargo portadora de un cierto riesgo que variará en intensidad en función a la manera en la que se lleva a cabo.

* La autora desea agradecer al Consejo de Investigación en Ciencias Humanas de Canadá (CRSH), al Centro de Investigación para el Desarrollo Internacional de Canadá (CRDI) así como a la Fundación Desjardins que financiaron estas investigaciones.

** Profesora de la Facultad de derecho de la Universidad de Sherbrooke (Canadá). LL.B. (McGill University), B.C.L. (McGill University), LL.M. (Université Laval), Doctorado en derecho (Université de Bordeaux), LL.D. (Université Laval). Correo electrónico: Marie-Claude.Desjardins4@USherbrooke.ca 
Palabras clave: comercio justo - derecho estatal - normas privadas derechos de los trabajadores - protección del medio ambiente

\title{
INTERACTIONS BETWEEN STATE LAW AND FAIRTRADE CERTIFICATION: A BETTER PROTECTION GUARANTEE FOR WORKERS' RIGHTS AND ENVIRONMENT
}

\begin{abstract}
The Fairtrade certification is an interesting Corporate social responsibility (CSR) model for its interactions with State law because its provisions and the way it operates promote a better application of it. The reference to State law is a means to incorporate State law into the regulatory body of Fairtrade certification. The reiteration of the obligation of producers to respect national legislation also constitutes a way to ensure that Fairtrade certification does not cause a level down to the producers demands. The reference to national legislation and practices allows, conversely, does not impose disproportionate obligations to producers. By insisting on the necessity of conforming standards from emerging standards rules to those who come of Law State, , it also allows a better understanding of law state. Despite these advantages, there is a certain risk related to the incorporation of national law, which varies in intensity depending on the way it is conducted.
\end{abstract}

Key words: Fairtrade - State law - private standards- rights of workers - environmental protection

\section{INTRODUCCIÓN}

Aunque surgidas a menudo de entidades privadas, como las empresas y las organizaciones no gubernamentales, los códigos de conducta y las certificaciones de responsabilidad social de la empresa (RSE) no están del todo desprovistas de una relación con el derecho. En varios puntos, mantienen una relación con el derecho en vigor en los países donde toman vida. En efecto, numerosos instrumentos de RSE hacen referencia, piden prestado textualmente, reformulan o se inspiran en las normas que se originaron de convenios internacionales (Daugareilh, 2008). Estos interactúan también con el derecho nacional. Es este último aspecto el que nos interesa particularmente en el marco del presente artículo. 
Aunque sea posible pretender que un régimen de certificación constituye un ordenamiento jurídico en sí mismo (Desjardins, 2015), este último no puede hacer abstracción del orden jurídico estatal en el cual se encuentra. En efecto, desde un punto de vista jurídico, es necesario comprender que una empresa no tiene por qué elegir entre el respeto a las disposiciones de derecho estatal o de aquellas de un instrumento de RSE. El orden jurídico estatal siendo obligatorio, si decide conformarse a las normas decretadas por un instrumento de RSE, no puede sin embargo renunciar a conformarse a las normas provenientes del derecho estatal. Así pues, teóricamente, las normas previstas en el código de conducta deberían ser respetadas además de las de derecho estatal. Es en efecto, lo que considera la Unión Europea en su definición de RSE: "RSE es la integración voluntaria de las consideraciones medio ambientales y sociales en las actividades empresariales, además de las exigencias jurídicas y las obligaciones contractuales" (Parlamento Europeo, 2007). Ahora bien, en realidad, no siempre es el caso. Puesto que la aplicación del derecho estatal es, en numerosos países, más o menos controlada y que los recursos en justicia son concretamente difíciles de acceso para la mayoría de los trabajadores (Javillier, 2008), la adopción de un código de conducta puede generar un beneficio doble para una empresa que estuviera más interesada por las ganancias que por el bienestar de sus trabajadores y la protección del medio ambiente. Esta consigue dar brillo a su reputación respetando al mismo tiempo obligaciones menos exigentes que aquellas previstas por el derecho estatal. Se trata entonces, en este caso, de una forma de excusa con respecto al derecho estatal. Además de apaciguar las críticas de los consumidores y de las organizaciones no gubernamentales $(\mathrm{ONG})$, estas iniciativas pueden tener como consecuencia el desviar la atención del Estado hacia empresas cuyas prácticas parecen más sospechosas. Afortunadamente, no es el caso de todos los instrumentos de RSE ni de todas las empresas implicadas en una gestión de RSE. Una pregunta se plantea entonces: ¿Cómo asegurarse de una interacción adecuada entre un instrumento de RSE y el derecho estatal?

En el marco de este artículo presentaremos un modelo de certificación de RSE interesante por su interacción con el derecho estatal: el régimen de certificación Comercio Justo Fairtrade International ${ }^{1}$. Al término de un estudio profundo en el ámbito del vino justo realizado en África del Sur,

1 La certificación Comercio Justo de la que se trata en este artículo es aquella denominada Fairtrade International Disponible en $<$ http://www.fairtrade.net $>$ y $<$ http://www.flo-cert.net/>. Este régimen está dividido en dos organizaciones. Fairtrade International (FLO) y FLO-Cert. FLO es responsable de la edición de los estándares y del apoyo a productores mientras que FLO-Cert es responsable de la certificación y de la inspección. 
en Chile y en Argentina, llegamos a la conclusión de que sus disposiciones y su funcionamiento favorecen claramente una mejor aplicación del derecho estatal. Los resultados presentados en este artículo se basan en investigaciones realizadas dentro del sector del vino Fairtrade. Nuestras investigaciones involucraron investigaciones de campo en los tres principales países productores de vino Fairtrade que son Argentina, Chile y Sudáfrica. Se realizaron más de 80 entrevistas personales semiestructuradas con trabajadores agrícolas, productores, funcionarios gubernamentales, organizaciones no gubernamentales, investigadores académicos, sindicatos, etc. Además se realizaron observaciones participantes.

\section{EL RÉGIMEN FAIRTRADE INTERNACIONAL(FLO)}

Antes de entrar verdaderamente en el corazón de nuestro análisis, proporcionemos primero algunas precisiones sobre nuestro sujeto de estudio, es decir, la certificación Comercio Justo Fairtrade International (FLO). El FLO es un organismo creado en 1997 y cuyo principal objetivo consistía en reunir las iniciativas nacionales de certificación Comercio Justo como Max Havelaar et Transfair. El régimen FLO es el régimen de certificación de comercio justo más difundido y conocido a través del planeta, agrupa 1,3 millones de agricultores y de trabajadores agrícolas repartidos en las 1149 organizaciones certificadas que se encuentran en 70 países. Las ventas de productos certificados comercio justo para FLO estaban evaluadas en 2012 en 4,8 mil millones de euros (Fairtrade International, 2013). Se cuenta hoy con más de 20 categorías de productos ${ }^{2}$ por los cuales es posible obtener una certificación FLO. Este régimen se divide en dos organizaciones: Fairtrade International (FLO) y FLO-Cert. FLO es responsable de la especificación de los estándares y del apoyo a los productores mientras que FLO-Cert es responsable de la certificación y de las inspecciones.

La certificación FLO reposa sobre un conjunto de normas consignadas en documentos llamados estándares. Para los productores, estos estándares se dividen en dos grandes categorías: los estándares genéricos y los estándares específicos. Los estándares genéricos son, como su nombre lo indica, estándares aplicables a todos y sin importar el tipo de productos cultivados y sin importar tampoco su país de

2 En 2015, los siguientes productos podían obtener una certificación FLO: plátano o banana, café, cacao, frutas secas, frutas frescas, legumbres frescas, jugo de frutas, hierbas y especias, miel, nueces y granos oleaginosos, cereales (fonio, quínoa, arroz), azúcar de caña, té, uvas para vino, oro, flores y plantas, fibra vegetal, balones deportivos, madera y granos de soya y legumbres secas. FLO (2014). 
producción. Sin embargo, una distinción se impone en cuanto al tipo de empresa o de organización productora. Así, existen tres tipos de estándares genéricos: aquellos destinados a una organización de pequeños productores organizada, aquellos aplicables a una organización de pequeños productores aún no organizada democráticamente y aquellos previstos para una empresa que depende de una mano de obra asalariada. Además de los estándares genéricos, las normas del régimen Comercio Justo Fairtrade se encuentran entre otros en los estándares específicos al producto.

\section{DIFERENTES FORMAS DE INTERACCIONES}

Contrariamente a numerosos códigos de conducta y otros instrumentos de RSE, los estándares de la certificación FLO no se contentan con referirse a las normas internacionales (Dillier, 1999), estos conceden igualmente un lugar importante a la legislación nacional que debe ser respetada a menos que esta última se halle en conflicto con los estándares o el derecho internacional, en cuyo caso la obligación más exigente es la que prevalece:

Fairtrade International exige que las empresas cumplan siempre con la legislación nacional, a menos que esa legislación esté en conflicto con las normas y convenciones internacionalmente reconocidas, en ese caso prevalecerán los criterios más elevados. Sin embargo, si la legislación nacional establece normas más elevadas o garantiza a los trabajadores condiciones más favorables que Fairtrade International en algún aspecto, entonces será la legislación nacional la que prevalecerá. Se aplica el mismo principio para las prácticas regionales o de sectores específicos ${ }^{3}$.

Es interesante tener en cuenta que los estándares FLO no se limitan a la legislación para determinar lo que constituye el corpus normativo de una región, pero que estos conceden igualmente una atención particular a las prácticas. Esto demuestra una apertura interesante por parte de FLO puesto que opta por un enfoque pluralista de la concepción del derecho y sus fuentes, que toma en cuenta aún más la realidad, la cual es a veces ampliamente diferente del derecho sobre papel.

Aunque el principal problema al cual se enfrenta el derecho en ciertos países sea la aplicación incompleta de la legislación, sucede también a 
veces que esta última es vetusta y por consecuencia no sea conforme a la realidad. Precisando así, que las prácticas pueden ser consideradas como representando el estándar mínimo, se remedian hasta cierto punto las debilidades de estas leyes inadaptadas.

El hecho de referirse a la práctica también ayuda a superar la ausencia de disposiciones legislativas. Por ejemplo, cuando no existe un salario mínimo legal, FLO se refiere al salario nacional o regional medio por un empleo similar.

Incluso si no lo mencionamos explícitamente en este artículo, las convenciones colectivas concluidas por el sector, cuando existen, son igualmente consideradas a fin de determinar las exigencias aplicables a los productores. Lo que nos lleva a concluir algunos artículos más específicos que lo refieran. El artículo 3.5.1 del Criterio de Comercio Justo Fairtrade para trabajo contratado, entre otros, es instructivo sobre la interpretación que conviene proporcionar a la disposición que impone la conformidad al derecho nacional: "Su empresa debe establecer los salarios de los trabajadores y otras condiciones de empleo de acuerdo a las regulaciones legales o del Convenio de Negociación Colectiva si éste existe o al salario medio regional o al salario mínimo oficial para empleos similares, lo que sea más alto, con la intención de aumentar continuamente los salarios."

Este artículo muestra en efecto que la legislación, las prácticas y el convenio colectivo celebrados para el sector deben servir al establecimiento de las condiciones de trabajo que serán concedidas a los trabajadores. Así pues, además de esta disposición general que exige el respeto de la legislación nacional en vigor, otras disposiciones más específicas establecen igualmente relaciones de interpenetración con la legislación nacional.

Se indica que la legislación en vigor y las prácticas que están actualmente en curso constituyen el punto de referencia para la evaluación de ciertas obligaciones, en particular en lo que respecta al salario $^{4}$, al número de semanas de permiso de maternidad ${ }^{5}$, al número de

4Art. 3.5.1 Criterio de Comercio Justo Fairtrade para Trabajo Contratado : « Su empresa debe establecer los salarios de los trabajadores y otras condiciones de empleo de acuerdo a las regulaciones legales o del Convenio de Negociación Colectiva si este existe o al salario medio regional o al salario mínimo oficial para empleos similares, el que sea más alto, con la intención de aumentar continuamente los salarios ».

5 Art. 3.5.15 Criterio de Comercio Justo Fairtrade para Trabajo Contratado : « Su empresa debe garantizar a sus trabajadores al menos 8 semanas de licencia de maternidad con el pago que establezcan las leyes nacionales y nunca inferior a $2 / 3$ del pago regular, el que sea mayor, sin incluir en este período las vacaciones anuales y sin perder ningún privilegio a causa de este permiso. Su empresa no debe poner final contrato de 
horas regulares trabajadas por semana ${ }^{6}$, a los descansos anuales ${ }^{7}, \mathrm{y}$ a las cotizaciones de seguridad social a pagar ${ }^{8}$.

Ella puede también servir para justificar ciertas adaptaciones regionales de los estándares FLO, como por ejemplo, el hecho de quemar los residuos orgánicos en vez de reutilizarlos:

Usted debe dar a conocer a sus miembros la reutilización de desechos orgánicos a través de la implementación de prácticas que permitan el reciclaje de nutrientes. Usted y los miembros solo pueden quemar los desechos orgánicos si es requerido por la legislación aplicable con fines fitosanitarios, o si es una práctica evidentemente más sostenible".

\section{RAZÓN DE SER Y UTILIDAD DE ESTAS INTERACCIONES}

Pero ¿para qué sirve la referencia al derecho nacional en los estándares FLO? Esta pregunta es mucho más importante cuando recordamos que a diferencia de las convenciones internacionales que deben ser ratificadas y en algunos casos, transpuestas en el orden jurídico nacional a fin de ser creadoras de derechos y deberes para los ciudadanos, el derecho nacional es directa y automáticamente aplicable a cada uno. Si la referencia a las normas internacionales dentro de los estándares de la certificación están justificadas por el objetivo de llenar las lagunas de ciertos Estados, es más difícil comprender el por qué se ha juzgado necesario y apropiado reiterar el respeto de la legislación nacional en una disposición de los estándares.

una mujer durante su embarazo o permiso de maternidad, excepto por causas no relacionadas con el embarazo o nacimiento del niño. La legislación nacional debe cumplirse si excede este requisito. »; Art. 3.3.20 Criterio de Comercio Justo Fairtrade para Organizaciones de Pequeños Productores: « Usted y los miembros de su organización deben fijar los permisos de maternidad, las prestaciones de seguridad social y los beneficios de carácter no obligatorio de acuerdo con las leyes nacionales o de acuerdo con la normativa del CCT cuando existan, o de acuerdo al convenio firmado entre la organización de trabajadores y el empleador, la que sea más favorable para el trabajador. »

6 Art. 3.5.9 Criterio de Comercio Justo Fairtrade para Trabajo Contratado ; Art. 3.5.12 Criterio de Comercio Justo Fairtrade para Trabajo Contratado : « Su empresa debe compensar las horas extras con un suplemento. El suplemento debe ser pagado según un factor de 1.5 para el trabajo realizado en un día de trabajo normal y pagado según un factor de 2 para el trabajo realizado en el día regional de descanso, en los festivos nacionales y para el trabajo nocturno, a menos que se defina otra cosa en la legislación nacional, en el Convenio de Negociación Colectivo en los acuerdos con los sindicatos ».

7Art. 3.5.13 Criterio de Comercio Justo Fairtrade para Trabajo Contratado: « Su empresa debe garantizar a los trabajadores al menos 2 semanas de vacaciones pagadas al año, sin incluir en ellas los permisos ocasionales y por enfermedad. El período de permiso anual debe ser acorde la legislación nacional y/o a lo detallado en el convenio de negociación colectiva específico o del sector, si alguno de ellos supera las dos semanas. »).

8 Art. 3.5.19 Criterio de Comercio Justo Fairtrade para Trabajo Contratado: « Su empresa debe proporcionar seguridad social legal a todos los trabajadores. » ; Art. 3.3.20 Criterio de Comercio Justo Fairtrade para Organizaciones de Pequeños Productores.

9Art 3.2.31 Criterio de Comercio Justo Fairtrade para Organizaciones de Pequeños Productores. 
Esta referencia al derecho estatal nos parece primero y ante todo como un medio de incorporar el derecho estatal al corpus normativo de la certificación Comercio Justo Fairtrade. Precisando a los productores que están sumisos a la ley estatal en los estándares del Comercio Justo Fairtrade, la obligación de respetar este corpus normativo integra por lo tanto el ordenamiento jurídico Comercio Justo Fairtrade proporciona así una base sobre la cual fundar el derecho de ejercer un control del respeto de estas normas para los productores. En efecto, sin esta disposición, los inspectores de FLO-Cert podrían difícilmente justificar una intervención con el fin de obligar a las empresas certificadas a respetar las disposiciones previstas por la legislación nacional, que serían más exigentes que los estándares de la certificación, porque esta última pertenecería a un orden jurídico externo y a aquel de donde emana su jurisdicción. El hecho de que los inspectores del régimen Comercio Justo Fairtrade puedan intervenir para hacer respetar las normas de derecho nacional favorece indudablemente la implantación de este último, sobre todo en los países en donde las inspecciones estatales son poco frecuentes o inexistentes.

Esta integración de normas de derecho nacional en el seno del régimen Comercio Justo Fairtrade permite igualmente a los individuos, víctimas de la violación de las disposiciones de derecho por sus empleadores, exigir el respeto por una vía alterna diferente a los tribunales estatales. Esto puede revelarse interesante para los trabajadores que no tienen generalmente un acceso a estos tribunales por falta de medios y de conocimientos, en razón de una distancia demasiado grande que tienen que recorrer para ir y llegar o de un riesgo muy elevado de represalias, pero también en el caso donde el acceso les es negado por motivos discriminatorios arbitrarios (Khan, 2010).

La reiteración de la obligación que tienen los productores de respetar la legislación nacional constituye igualmente un medio de asegurar que la certificación Comercio Justo Fairtrade no tenga como consecuencia una nivelación a la baja de las exigencias aplicables a los productores, ya sea que provengan de la legislación estatal o del régimen Comercio Justo Fairtrade. Al reconocer que pueden existir diferencias entre lo que prevé el derecho estatal y el régimen Comercio Justo Fairtrade, se establece una clara distinción entre estos dos regímenes lo que tiene por efecto reducir el riesgo de confusión.

Se trata igualmente de un recordatorio a las partes constitutivas del sistema jurídico Comercio Justo Fairtrade de que la conformidad con las 
disposiciones de uno no equivale necesariamente a la conformidad del otro. Contrario a la referencia a las normas internacionales, no se trata aquí de remediar la falta de normas elaboradas por el Estado o su debilidad, se trata más bien, de asegurar que los puntos fuertes de la legislación en vigor no sean desatendidos o desviados debido a la existencia de la certificación Comercio Justo Fairtrade.

Las disposiciones relativas al número de horas de una semana de trabajo normal, constituyen un buen ejemplo a fin de explicar nuestro propósito. El artículo 3.5.9 del Criterio de Comercio Justo Fairtrade para Trabajo Contratado establece que son 48 horas el tiempo de trabajo semanal regular. Ahora bien, la legislación chilena redujo, en enero de 2005, el número de horas de 48 a 45 (Dirección del trabajo, 2004). Si los estándares de la certificación Comercio Justo Fairtrade hicieran caso omiso de la ley nacional, los inspectores de FLO-Cert podrían conceder la certificación a una empresa chilena aún si esta acordara a sus empleados una tasa horario aumentada (correspondiente a la de horas extras) que a partir de la 49ava hora de trabajo por semana, lo que sería contrario a la legislación chilena pero conforme a los estándares FLO. En teoría, la obligación de pagar un salario por hora más elevado a partir de la 46ava hora de trabajo se mantendría respecto al productor chileno certificado puesto que no es porque integra el régimen Comercio Justo Fairtrade que no esté ya vinculado a las obligaciones que se derivan al pertenecer al orden jurídico nacional. En efecto, la pertenencia a uno no excluye el vínculo al otro. Ahora bien, en realidad, dado que los controles estatales son poco frecuentes y que los productores tienen a menudo un escaso conocimiento de la ley, sobre todo cuando esta ha sido recientemente modificada, si FLO se contentara de exigir al productor el pago de horas extras después de la 48ava hora trabajada de la semana, es, en la mayoría de los casos, la regla que prevalecería. El hecho de no tomar en cuenta la legislación nacional tendría a veces pues como efecto, la disminución de las exigencias impuestas por esta última.

Al insistir sobre la necesidad de deber conformarse tanto a las normas surgidas de los estándares como a aquellas que provienen del derecho estatal, se favorece igualmente un mejor conocimiento del derecho estatal. Los productores no pueden contentarse con seguir las disposiciones que se encuentran en los estándares FLO, deben emprender un aprendizaje de su propio derecho, lo que representa una nueva tarea para varios de ellos. Es por otra parte, lo que nos han confiado numerosos productores interrogados. Varios nos confesaron que jamás habían tomado verdaderamente el tiempo de conocer las 
exigencias legales sobre ciertos aspectos de la relación empleadoempleador. Uno de ellos admitió por otra parte haberse equivocado siempre en lo que concierne a la manera en que la ley exige que se proceda con la contabilización de los días de enfermedad antes de formar parte de un régimen de Comercio Justo Fairtrade. Ellos procedían como mejor podían con sus conocimientos y siguiendo una cierta lógica. Ahora bien, después de su integración al régimen Comercio Justo Fairtrade, conocen más su propio derecho nacional y están en la medida de ponerlo en obra de una manera más adecuada que de lo que lo hacían anteriormente. Este punto destacado de la certificación Comercio Justo Fairtrade es para señalarse ya que permite responder a las críticas al efecto de que a partir del momento en el que la certificación Comercio Justo Fairtrade no está más en vigor en una empresa, todos los beneficios anteriormente obtenidos desaparecen. En efecto, cuando la empresa ya no es más el objeto de inspecciones FLO-Cert existe un riesgo de que los dirigentes de esta última tengan una actitud más relajada en cuanto a la conformidad de su práctica con relación al derecho, pero el conocimiento de las normas de derecho estatal adquiridas en el momento de pertenecer al régimen Comercio Justo Fairtrade permanecen inevitablemente.

Si la referencia a la legislación y a las prácticas nacionales o regionales permite evitar la imposición de una exigencia demasiado débil en relación a aquellas con las cuales los productores se encuentran ya sumisos, en virtud del derecho estatal, su consideración de las especificidades del lugar de producción permite, inversamente, no imponer tampoco obligaciones desproporcionadas a los productores.

El objetivo de la certificación Comercio Justo Fairtrade es en efecto el de mejorar las prácticas de los agricultores en materia de medio ambiente y en consideración a sus empleados. Ahora bien, aunque se contempla la rectificación de las exigencias impuestas a los productores agrícolas en estas materias, una aumentación demasiado drástica de estas, podría resultar fatal para algunos. Es lo que podría producirse si fijáramos, por ejemplo, un salario mínimo uniforme dentro de los estándares genéricos. Ya que estamos aquí, recordemos que los estándares genéricos son aplicables a todos los productores que han obtenido la certificación Comercio Justo Fairtrade sin importar el país de producción, el producto cultivado o fabricado, el índice de desarrollo, las condiciones climáticas o el ingreso anual por habitante de su país. Aunque los países contemplados por la certificación Comercio Justo Fairtrade estén todos considerados "en desarrollo" por FLO, hace falta reconocer que no están 
todos en el mismo nivel y que no comparten todos las mismas características. Como ejemplo, Chile y Argentina forman parte de los países que cuentan con un índice de desarrollo humano muy elevado según la clasificación de 2013 mientras que se atribuyó a Pakistán y a Chad un índice de desarrollo bajo (Programa de las Naciones Unidas para el desarrollo, 2013). Habida cuenta de esta clasificación, por ejemplo, es probable que un agricultor chadiano, no esté en condiciones de ofrecer el mismo salario-horario a sus empleados que un agricultor chileno. Ahora bien, si se impusiera un salario mínimo único dentro de los estándares genéricos y que fuera suficientemente elevado para el trabajador chileno, nos encontraríamos muy probablemente imponiendo al granjero chadiano una obligación desproporcionada. La imposición de las exigencias uniformes a todos los productores, cualesquiera que fuera su país, no está entonces indicada ya que esta podría amenazar seriamente la existencia de ciertas empresas y asociaciones de productores. Es entonces lógico y del todo deseable que ciertas disposiciones tengan en cuenta estas disparidades. Para hacer esto, FLO habría podido redactar estándares que variarían según el país de producción y el producto cultivado, lo que habría sido una tarea gigantesca y cuyos resultados no podrían ser otra cosa que imperfectos. FLO ha optado por un enfoque más realista y más eficaz en lo que se refiere a la legislación estatal.

Por el contrario, la referencia a la legislación no debe tampoco servir de medio para reducir en exceso las obligaciones impuestas a los productores. Eso sería por ejemplo el caso de una legislación incompleta, inexistente o cuyo contenido no haya sido actualizado desde hace mucho tiempo. FLO en consecuencia, debió imponer un cierto mínimo el cual no es posible derogar a pesar de la referencia a la legislación estatal, una de las disposiciones del Criterio de Comercio Justo Fairtrade para Trabajo Contratado relativa a las horas extras suplementarias es un buen ejemplo:

Su empresa debe cumplir con la legislación nacional y local aplicable y los estándares del sector en cuanto a las horas de trabajo y las regulaciones sobre horas extras. Su empresa no debe exigir a los trabajadores que trabajen más de 48 horas a la semana periódicamente ${ }^{10}$.

A la luz de estas constataciones, concluimos que, dado que intenta remediar las debilidades y lagunas de los estados no tanto en cuanto al 
contenido normativo de su orden jurídico, sino más bien sobre el de la aplicación y que ésta consiste en reafirmar el derecho estatal más que competir contra él con normas más vagas y menos obligatorias, la integración de la legislación estatal en el seno del régimen FLO es una buena cosa.

Tomando como base la legislación estatal, FLO se asegura igualmente de una mayor legitimidad de sus normas, dado que se consideran haber sido el resultado de un proceso democrático. A este sujeto, recordemos que una de las principales debilidades de la certificación Comercio Justo Fairtrade con respecto a los trabajadores es su déficit democrático. No son en efecto considerados como parte del proceso de elaboración de los estándares sino como simples beneficiarios. El derecho estatal, aunque sea imperfecto, incluye la característica de ser el resultado de un proceso democrático, que en principio, debería haber integrado, probablemente de manera indirecta, en efecto, a los trabajadores y toma en cuenta sus reivindicaciones. La integración de tales normas en el régimen Comercio Justo Fairtrade permite indudablemente una mejor aceptación de estas, lo que sería menos evidente si ellas provinieran de un régimen completamente desconocido del cual los trabajadores no formaran parte. Aquí conviene sin embargo señalar que FLO tendría una ventaja si integrara a los trabajadores en el proceso de la elaboración y revisión de las normas.

\section{RIESGOS DERIVADOS DE ESTAS INTERACCIONES}

Tal y como ocurre con la incorporación de las normas de derecho internacional, la incorporación de normas de derecho nacional es igualmente portadora de un cierto riesgo (Gravel, 2008) que variará en intensidad en función a la manera en la que se lleva a cabo. La integración de una norma procedente de un primer orden jurídico hacia un nuevo orden jurídico es susceptible de aportar modificaciones a esta norma inicial, lo que puede ser fuente de confusión, sobretodo, cuando los destinatarios son los mismos y que la aplicación de las normas es incompleta en el orden de origen. Poner entre las manos de entidades privadas el contenido normativo de derecho nacional constituye igualmente un factor de acentuación de este riesgo. Aunque las disposiciones de las cuales se trata sean relativamente simples y poco sujetas a interpretación múltiple, sigue siendo probable que los inspectores de Flo-Cert interpreten algunas de entre ellas de manera no del todo conforme o incompleta en relación a lo que hubieran hecho los jueces estatales en iguales circunstancias. Ahora bien, a pesar de este 
riesgo, opinamos que a pesar de todo en este contexto, se hace aún más necesario adoptar un enfoque realista más que purista. En otros términos, de dos males, es necesario escoger el menor. Uno de los problemas más escandalosos de la producción agrícola es el mantenimiento de miles de trabajadores en situación de explotación y de sus condiciones de vida miserables así como la destrucción del medio ambiente y de su biodiversidad a una intensidad y un ritmo alarmantes del cual es de sobra responsable la aplicación incompleta de las disposiciones legislativas. ¿Es necesario poner a un lado una herramienta susceptible de mejorar la aplicación del derecho del trabajo y del medio ambiente bajo pretexto de que hay un riesgo de confusión en cuanto al sentido que se debe dar a algunas nomas de este último? En ciertos casos la respuesta que debe darse a esta pregunta debería ser afirmativa. Ahora bien, en el caso de la certificación Comercio Justo Fairtrade pensamos que sería absurdo desalentar el recurso a un tal instrumento habída cuenta del hecho de que está dirigida a un número mínimo de individuos del orden jurídico estatal lo que puede ser poco susceptible de tener un impacto mayor en la interpretación dentro de las disposiciones en el orden jurídico estatal. (Bahdi, 2002-2003; Benyeklef, 2011; Slaughter, 2003)

A este efecto, puede hacerse un paralelo interesante con el uso de derecho estatal permitido al árbitro en el derecho canadiense. El árbitro aunque teniendo como principal instrumento de análisis el convenio colectivo firmado entre las partes en desacuerdo, también tiene a menudo recurso de los instrumentos jurídicos de derecho estatal para ayudarlo a resolver un litigio y justificar su decisión. Hecho interesante en esta utilización del derecho estatal, es que se le reconoce la posibilidad de adaptar, en una cierta medida, ciertas disposiciones de derecho o de principios jurídicos a fin de promover la paz industrial. Esta adaptación no puede en absoluto conducir a una interpretación poco razonable de estos pero un margen de maniobra creativo le está claramente permitido. Es lo que reconocieron numerosos tribunales canadienses, incluido entre ellos la Suprema Corte de Canadá que ha tenido recientemente que resolver un litigio, en un contexto de arbitraje, referente a una interpretación de principio de common law de preclusión. Aún si la interpretación que hizo de este principio el árbitro, no era del todo idéntica a la interpretación jurídica, es decir, aquella que hacen generalmente los tribunales judiciales canadienses, la Suprema Corte no invalidó su decisión al término de la revisión judicial ${ }^{11}$.

11 Nor-Man Regional Health Authority Inc.c.Manitoba Association of Health Care Professionals (2011), para 5-7. 
Hay que reconocer que nuestra posición al respecto de la utilización del derecho estatal en el seno del régimen Comercio Justo Fairtrade es muy cercana a aquella de los tribunales canadienses en materia de arbitraje. Si el derecho canadiense permite a un árbitro una cierta flexibilidad en la interpretación de normas de derecho estatal, parece que nuestro razonamiento dentro del contexto de la certificación Comercio Justo Fairtrade no esté completamente desprovisto de sentido y que esta manera de proceder no constituya un riesgo inaceptable para el derecho estatal. La Suprema Corte de Canadá hace hincapié en el hecho de que esta flexibilidad permanezca limitada por balizas, a saber, el carácter razonable de la decisión, elemento en efecto más difícil de instaurar en el contexto de la certificación Comercio Justo Fairtrade ya que no están sometidos al control de los tribunales estatales. Ahora bien, una colaboración entre las autoridades estatales y los inspectores FLO-Cert constituiría en efecto un medio de evitar desviaciones o una interpretación totalmente inadecuada. FLO-Cert debería a este fin exigir de los inspectores que ellos consulten de vez en cuando a los representantes de los ministerios pertinentes (medio ambiente, trabajo, agricultura como mínimo) y/o a los inspectores estatales.

Finalmente, a pesar de su pertinencia y de lo bien fundado, un último punto merece ser abordado en el análisis de estas disposiciones teniendo por efecto integrar las normas de derecho estatal nacional en el seno del régimen Comercio Justo Fairtrade, sobre todo en lo que se refiere a la disposición a la cual hace referencia de manera general ${ }^{12}$. Esta última puede quedar dubitativa debido a su amplitud. Es difícil créer que la inspección de FLO-Cert esté en condiciones de comprobar la conformidad de los productores respecto a todas las disposiciones del derecho estatal en cada uno de los 70 países en los cuales pueden ser certificadas las empresas y las asociaciones de productores. Es, por otra parte, lo que nos permitieron constatar nuestras investigaciones de campo. El tratamiento que habitualmente le es reservado por FLO-Cert en la práctica, consiste en limitar el derecho nacional al cual se hace referencia al que se remite a los sectores de las actividades conexas a las normas de FLO. En efecto no es raro que FLO-Cert haga referencia a las

12 «Fairtrade International exige que las empresas cumplan siempre con la legislación nacional, a menos que esa legislación esté en conflicto con las normas y convenciones internacionalmente reconocidas, en ese caso prevalecerán los criterios más elevados. Sin embargo, si la legislación nacional establece normas más elevadas o garantiza a los trabajadores condiciones más favorables que Fairtrade International en algún aspecto, entonces será la legislación nacional la que prevalecerá. Se aplica el mismo principio para las prácticas regionales o de sectores específicos. » Preámbulo Criterio de Comercio Justo Fairtrade para Trabajo Contratado. 
disposiciones de las convenciones colectivas aplicables a los sectores de las actividades o a la legislación laboral o medio ambiental en el informe de inspección con el fin de condenar ciertas prácticas o de declararlas de conformidad, o no, con el derecho estatal. A pesar de esta explicación, una cuestión permanece: ¿Por qué haber elegido una formulación tan inclusiva? Sugerimos que se trata de una manera para FLO de dejar la puerta abierta para situaciones eventuales de violación del derecho estatal en otros sectores que aquellos que hacen el objeto de los estándares.

Estos otros ámbitos del derecho no son objeto de una inspección por parte de FLO-Cert pero en la eventualidad donde el propietario de una empresa agrícola certificada por FLO fuera reconocido culpable, por ejemplo, en el marco de sus funciones, de fraudes en el seno de su empresa, este artículo permitiría a FLO dar por terminada la certificación. Esta interpretación nos parece ser avalada por el artículo 10.6 del contrato de licencia:

Grounds considered as « serious » may justify the immediate termination of the Contract by FLO. FLO shall notify the Licensee thereof by means of a recorded delivery letter with acknowledgement of receipt. « Serious grounds » may be : [...] Any criminal conviction that can affect the image of the LI or FLO.

\section{CONCLUSIÓN}

Resulta del análisis del contenido de los estándares de la certificación Comercio Justo Fairtrade que la integración y la referencia a las normas internacionales y nacionales procedentes del ordenamiento jurídico estatal, tal como fueron hechas en el régimen Comercio Justo Fairtrade, le confiere una mayor legitimidad y favorece un mejor conocimiento del derecho estatal y una mayor aplicación. La utilización que se hizo, demuestra claramente que el régimen FLO no tiene como vocación sustituir los instrumentos estatales jurídicos internacionales y nacionales lo que no sería por otro lado deseable sino por lo contrario, a apoyar su aplicación.

\section{REFERENCIAS BIBLIOGRÁFICAS}

Bahdi, Reem (2002-2003). Globalization of Judgment : Transjudicialism and the Five Faces of International Law in Domestic Courts, 34 Georgetown Washington International Law Review 555. 
Benyeklef, Karim (2011). Vers une gouvernance globale : émergence d'un droit postnational ?, dans Louise LALONDE et Stéphane BERNATCHEZ, La place du droit dans la nouvelle gouvernance étatique, Sherbrooke, Ed. Revue de droit de l'Université de Sherbrooke.

Daugareilh, Isabelle (2008). Corporate norms on Corporate Social Responsability and international norms, en Javillier, Jean-Claude (edit.), Governance, International Law and Corporate Social Responsability (Ginebra, International Labour Organisation) pp. 63-78.

Desjardins, Marie-Claude (2015). La certification équitable depuis une perspective juridique. L'exemple du secteur viticole. Cowansville, Editions Yvon Blais.

Diller, Janelle (1999). Responsabilité sociale et mondialisation : qu'attendre des codes de conduite, des labels sociaux et des pratiques d'investissement?, 138 (2) Revue internationale du travail, 107.

Dirección del trabajo (2004). Nueva Jornada Laboral, Disponible en: $<$ http://www.dt.gob.cl/1601/w3-article-72784.html>

Fairtrade International (2013). Unlocking the Power, Annual report 2012-2013, Disponible en : <http://www.fairtrade.net/fileadmin/ user_upload/content/2009/resources/2012-13_AnnualReport_Fair tradēntl_web.pdf>

FLO (2014). List of all FLO Standards, Disponible en : http://www.fair trade.net/fileadmin/user_upload/content/2009/standards/documents/20 14-05-15_List_of_Fairtrade_Standards.pdf

Gravel, Eric (2008) «Is the appropriation of international labour standards by new actors replacing or complementing the ILO's traditional standards-related work ? » en International Institute for Labour Studies (edit.), Governance, International Law \& Corporate Social Responsability, Research Series 116, p. 79.

Javillier, Jean-Claude (2008). Corporate Social Responsibility and Law : Synergies are needed for Sustainable Development, en Javillier, JeanClaude (edit.), Governance, International Law and Corporate Social Responsability, ILO, Genève, p.44.

Khan, Irene (2010), Pauvres en droits, (Paris, Max Milo). 
Programa de la Naciones Unidas para el desarrollo (2013). Informe sobre Desarrollo Humano 2013, El ascenso del Sur: Progreso humano en un mundodiverso, Disponible en: $<$ http://hdr.undp.org/sites/default/ files/hdr2013_es_summary.pdfs.

Slaughter, Anne-Marie (2003). A Global Community of Courts, 44 Harvard International Law Journal 191

\section{Normas citadas}

Criterio de ComercioJusto Fairtrade para Organizaciones de Pequeños Productores (2011), Disponible en <http://www.fairtrade.net/ fileadmin/user_upload/content/2009/standards/documents/2014-0716_SP_SPO.pdf $>$

Criterio de ComercioJustoFairtrade para TrabajoContratado (2014), Disponible en <http://www.fairtrade.net/fileadmin/user_upload/ content/2009/standards/documents/generic-standards/2014-0716_SP_HL_FINAL.pdf $>$

Resolución del Parlamento Europeo, de 13 de marzo de 2007, sobre la responsabilidad social de las empresas: una nueva asociación (2006/2133(INI))

\section{Jurisprudencia citada}

Canadá: Nor-ManRegionalHealthAuthority Inc.c.Manitoba Association of Health Care Professionals, [2011] CSC 59. 